\title{
A Model for User-Oriented Data Provenance in Pipelined Scientific Workflows ${ }^{\star}$
}

\author{
Shawn Bowers ${ }^{1}$, Timothy McPhillips ${ }^{1}$, Bertram Ludäscher ${ }^{1,2}$, \\ Shirley Cohen ${ }^{3}$, and Susan B. Davidson ${ }^{3}$ \\ ${ }^{1}$ UC Davis Genome Center, University of California, Davis \\ ${ }^{2}$ Department of Computer Science, University of California, Davis \\ ${ }^{3}$ Computer and Information Science, University of Pennsylvania \\ \{sbowers, ludaesch, tmcphillips\}@ucdavis.edu \\ $\{$ shirleyc, susan\}@cis.upenn.edu
}

\begin{abstract}
Integrated provenance support promises to be a chief advantage of scientific workflow systems over script-based alternatives. While it is often recognized that information gathered during scientific workflow execution can be used automatically to increase fault tolerance (via checkpointing) and to optimize performance (by reusing intermediate data products in future runs), it is perhaps more significant that provenance information may also be used by scientists to reproduce results from earlier runs, to explain unexpected results, and to prepare results for publication. Current workflow systems offer little or no direct support for these "scientist-oriented" queries of provenance information. Indeed the use of advanced execution models in scientific workflows (e.g., process networks, which exhibit pipeline parallelism over streaming data) and failure to record certain fundamental events such as state resets of processes, can render existing provenance schemas useless for scientific applications of provenance. We develop a simple provenance model that is capable of supporting a wide range of scientific use cases even for complex models of computation such as process networks. Our approach reduces these use cases to database queries over event logs, and is capable of reconstructing complete data and invocation dependency graphs for a workflow run.
\end{abstract}

\section{Introduction}

The importance of provenance information in scientific data and workflow management is widely recognized, as witnessed, e.g., by specialized workshops [4], research projects [17, and surveys [3120] dedicated to this topic, and by investigations on foundations of data provenance for queries and transformations [51923. However, current scientific workflow systems still offer little or no support for queries of interest to the end-users of these systems, e.g., researchers in

* Work supported in part by SciDAC/SDM (DE-FC02-01ER25486), NSF/SEEK (DBI-0533368), and NSF/GEON (EAR-0225673). 


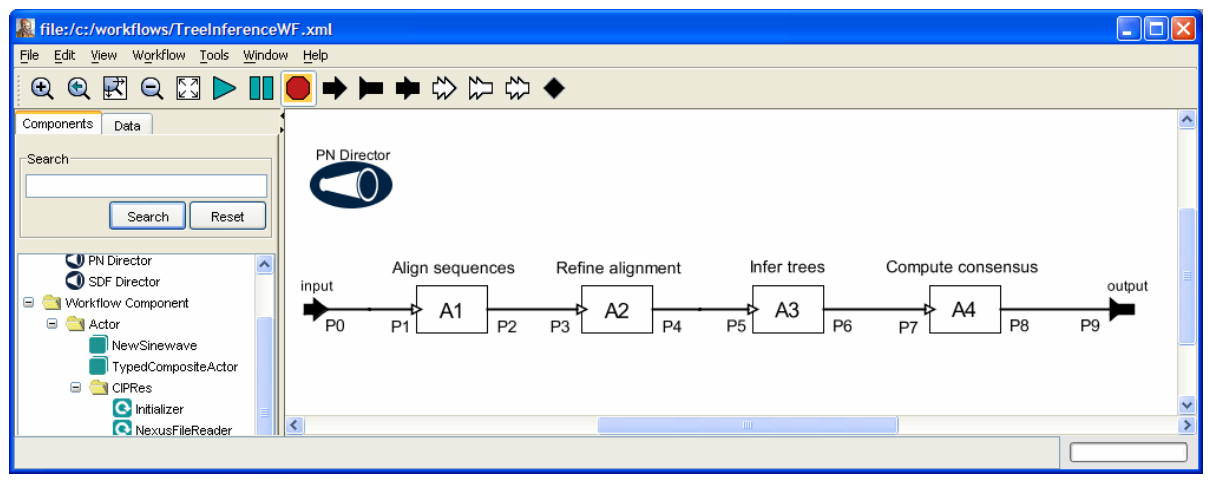

Fig. 1. A workflow for computing phylogenetic trees from input DNA sequences

the life or physical sciences. In this paper, we argue that concrete use cases, expressed in terms that are meaningful to the scientist, should drive the design of a provenance system. Moreover, such systems should be designed in terms of the models of computation $(\mathrm{MoC})$ that govern the execution of scientific workflows to ensure that all pertinent events are recorded in the execution log.

Fig. 11 shows an example workflow for inferring phylogenetic trees approximating the evolutionary relationships between organisms. DNA sequences for homologous genes from a number of taxa are provided as input to the workflow. Actor A1 performs an initial alignment of the sequences (e.g., using the program ClustalW 22]), and actor A2 refines this initial alignment (e.g., using Gblocks [7] 1] Actor A3 infers a set of phylogenetic trees from the aligned sequences (e.g., using DNAPARS [19]), and actor A4 computes the consensus of these trees (e.g., using CONSENSE [19]).

For such scientific workflows we would like to: (a) enable scientists to ask "scientific" questions about a workflow run by providing convenient queries against the run's execution log; and (b) have the system track the true data dependencies within a run so that answers to such scientific questions may be as accurate as possible. For example, the system should recognize independent "sub-runs" as such: The workflow in Fig. 1 may process multiple sets $s_{1}, s_{2}, \ldots$ of independent DNA sequences (e.g., corresponding to distinct genes in the taxa of interest) within a single workflow run $R$. In such cases, the system should not infer that the data products resulting from the different $s_{i}$ are interdependent. Rather, the system should answer accurately questions such as:

- Which phylogenetic trees were used to produce this consensus tree?

- Which DNA sequences does this consensus tree depend on?

- Which of the input DNA sequences were not used to derive any output consensus tree?

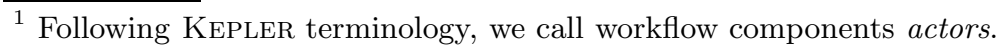


In this paper, we develop a provenance model designed to support such useroriented queries for pipelined models of computation where tracking data dependencies can be complex. For another example, consider an actor $A$ in an environmental monitoring workflow that computes a running average of temperature for each received measurement data token. Thus, upon each invocation or firing of $A$, the actor consumes a temperature token and emits a new running average token. To calculate the running average over multiple firings, $A$ must maintain state. For the provenance system this means that every produced data token must be recorded as dependent not just on those input tokens received since the last time the actor fired, but on all tokens received since $A$ was initialized. Conversely, if $A$ is to limit the running averages to readings taken on a particular day, then $A$ 's state is reset once per day. There are no dependencies between tokens produced after a reset and tokens consumed prior to the reset. This observation naturally partitions token streams, as well as actor firings, into semantically meaningful firing rounds 2 Clearly, a provenance system should be able to observe and record new rounds of firing to avoid reporting false dependencies.

The running average example described above illustrates a general property of scientific workflows implemented as process networks [12 15 13]: actors need not produce output tokens derived exclusively from tokens received since the last output token was produced. That is, actors in process networks do not generally compute functions on sets of consecutively received inputs. Rather, they may carry out arbitrarily complex transactions on streams of inputs, including running averages, filters, sliding windows, and iterative computations.

Capturing these transaction boundaries is essential for accurately recording scientific workflow provenance. In this paper, we show how this essential information can be represented in a simple tabular event log. Our approach is easy to implement, e.g., in the KEPLER scientific workflow system, where token-read and token-write events can be automatically captured by the workflow framework. Announcing a new round of firing (e.g., by signaling a reset event), on the other hand, is performed by actors themselves, which "know" when they are beginning an independent task (such as a "sub-run" $s_{i}$ above, or new daily average temperatures).

The rest of this paper is organized as follows. Section 2 briefly overviews scientific workflows within KEPLER, focussing on pipelined execution models. Section 3 presents our provenance model, which consists of read, write, and state reset events. We also describe in Section 3 how to compute data and actor dependency graphs (e.g., for computing data lineage) from corresponding event logs. Section 4 describes a set of operations (or views) over the provenance model for supporting "scientist-oriented" provenance queries. A number of examples are given, which define parameterized queries for the workflow of Figure 1. Finally, Section 5 summarizes our contributions and future work.

${ }^{2}$ A round is somewhat analogous to a database transaction, specifically in that it constitutes a logical unit of work. 


\section{Preliminaries}

\subsection{Workflow Graphs, Actors, and Tokens vs. Data Objects}

We adopt notions and terminology from KePLER, a scientific workflow system extending Ptolemy II. Workflows are composed by placing actors on a design canvas, and "wiring" them together to form the desired workflow graph (Fig. (1). Actors communicate through their input and output ports. In a workflow graph $W$, output ports can be connected to input ports, establishing unidirectional dataflow channels. Actors communicate through these channels by passing tokens.

By default tokens are immutable and "disposable", i.e., every token $t$ is written only once [15] and thus lives only between its creation on an output port, and its consumption at subsequent input ports. Thus, even if an actor passes on a data object unchanged, a new token-id is created, facilitating tracking of token dependencies. A separate object-id is used to track object dependencies. By object $(t)$ we denote the data object represented by the token $t$. To support user-oriented queries, we associate with an object $o$ one or more types types $(o)$.

The ports of an actor $A$ are denoted ports $(A)$. We assume that port-ids are globally unique, i.e., they include a unique actor-occurrence-id and a port-name which is unique to the actor occurrence. A port is either an input or output, so $\operatorname{ports}(A)=i n(A) \dot{\cup} \operatorname{out}(A)$. Some input ports $\operatorname{pars}(A) \subseteq i n(A)$ may be distinguished as parameters for configuring $A$ 's behavior. The signature $\Sigma_{W}:=$ $i n(W) \rightarrow \operatorname{out}(W)$ of a workflow $W$ is given by a set of distinguished inputs $\operatorname{in}(W)$ and outputs out $(W)$. As shown in Figure 1, the distinguished workflow input and output ports are connected to a subset of the input and output ports of the workflow's actors.

\subsection{Directors}

The model of computation (MoC) of a workflow is not defined by actors, but specified by a separate component called a director. Thus, KEPLER allows workflow designers to choose among different MoCs by choosing appropriate directors. A director specifies and (effectively) mediates all inter-actor communication, separating workflow scheduling and runtime orchestration (a director's concern) from individual actor execution (an actor's concern). This separation achieves a form of behavioral polymorphism [14], resulting in more reusable actor components. KEPLER provides a variety of directors that implement process network (PN and SDF), discrete event (DE), continuous time (CT), and finite state transducer (FST) semantics.

\subsection{Pipelined Execution}

In the process network MoC, the PN director executes each actor as a separate process (or thread). Channels are used to send and to buffer token streams 
between actors. Each actor can decide independently how many tokens to consume before writing out a number of output tokens. In this way, workflows that run using the $\mathrm{PN}$ director not only exhibit task parallelism, but also pipeline parallelism. For example, during a single workflow run, each actor in Figure 1 can execute multiple times, and different actors can execute concurrently.

A number of other MoCs can be considered as special cases of the basic process network model [1215. In the synchronous dataflow (SDF) model [13], actors a priori define fixed token consumption and production rates. This model allows the SDF director to statically schedule actors, while guaranteeing, e.g., that (unlike in the general PN case) deadlocks cannot occur and that buffers have a fixed size. By DAG (directed acyclic graph) we denote a MoC that is common in job-centric grid workflows 2110]: nodes represent jobs, and directed edges represent execution dependencies between jobs. Thus, a DAG director can simply execute the jobs in the partial order implied by the job dependency graph. This can be seen as a limited special case of SDF, with an acyclic workflow graph, actors having at most one input and one output port, consuming and producing a single token per workflow run, respectively, and in which each actor is invoked exactly once (unlike in the more general SDF or PN cases).

\section{A Provenance Model for Pipelined Workflows}

In this section we describe a provenance model that can handle the process network $(\mathrm{PN})$ model of computation, and thus specialized versions such as SDF and DAG as well. To execute a workflow (graph) $\mathcal{W}$, we must "bind" (i.e., select) input data $\mathbf{i}$ on which $\mathcal{W}$ will operate. Often $\mathcal{W}$ is also parameterized using initial parameter settings $\mathbf{p}$. It is customary to record identifiers for $\mathcal{W}, \mathbf{p}$, and $\mathbf{i}$ as part of the provenance information. Finally, a $\operatorname{MoC} M$ is needed (e.g., $\mathrm{PN}, \mathrm{SDF}, \mathrm{DAG})$ to determine how the workflow is executed 3 Taken together, the equation

$$
\mathbf{o}=M\left(\mathcal{W}_{\mathbf{p}}(\mathbf{i})\right)
$$

denotes a workflow execution in which the output o is obtained by applying a suitable model of computation $M$ to an appropriately instantiated workflow $\mathcal{W}$.

\subsection{Runs, Traces, and Observables}

Each MoC $M$ formally defines the notion of legal computations or runs, such that one can determine whether a particular run $R$ of a workflow $\mathcal{W}$ is a legal representation (w.r.t. $M)$ of an execution $\mathbf{o}=M\left(\mathcal{W}_{\mathbf{p}}(\mathbf{i})\right)$. A workflow trace $\mathcal{T}$ is an approximation of a run $R$, according to a model of provenance. As recorded by a provenance model, a trace approximates a run by recording functional and non-functional observables. For example, an SDF director precomputes a static workflow schedule (based on actor consumption and production rates),

${ }^{3}$ Some MoCs might also be aware of resources such as cluster (or grid) nodes and transport protocols, and schedule a distributed workflow accordingly. 
and using this schedule signals each actor to fire in turn. Thus, actor firings are directly observed in SDF. In contrast, the size of a token (or rather the object it represents) and the timestamp when the token was created are non-functional observables: according to the $\mathrm{MoC}$, the outcome does not depend on these. Nonfunctional observables can be useful to record, e.g., to benchmark actor execution times or data transfer times between actors, but are not essential for determining data dependencies 4

\subsection{The Read, Write, State-Reset (RWS) Provenance Model}

Here we consider a concrete model of provenance, called the RWS model, which records $\boldsymbol{r e a d}, \boldsymbol{w}$ rite, and state-reset events for each actor in a workflow run. These events are stored in a relational event log. This model focuses on only a minimal set of observables that allow us to answer many science-oriented user questions (see next section), while ignoring non-functional observables such as timestamps, although such information can be easily added. Figure 2 is an example of an event $\log$ for a run of the workflow given in Figure 1. During a workflow run, a read event is added to the event log each time an actor reads a token from a port. Similarly, a write event is added to the log each time an actor writes a token to a port. A series of reads followed by writes denotes an actor firing. Note that in a particular firing $F_{j}$, an actor may use data that it read in a previous firing $F_{i}$ to generate output (e.g., this is the typical behavior of a running-average actor, as described in Section 1). In this case, we say the actor maintains state across firings, and state-reset events denote when the state is "flushed" (reset). The firings between reset events constitute a firing round 5

As shown in Figure 2, each row in an event log contains: the location $E_{l o c}$ of the event, which is either a port (for read and write events) or an actor (for state-reset events); the event type $E_{t y p}$, which is either 'r' for read events, 'w' for write events, or ' $\mathrm{s}$ ' for state-reset events; the token identifier $E_{\text {tok }}$ that was read or written at the port (null for state-reset events); and a firing count $E_{\text {fire. }}$

Because actor port identifiers are unique across a workflow, and tokens are written once, the port and token identifiers recorded for each read and write event enable the reconstruction of the flow of data through the workflow run. However, these events alone are not sufficient to reconstruct data dependencies. We use the state-reset events (as described above) along with the firing count for this purpose. In particular, the firing count is incremented independently for each actor whenever (1) an actor switches from writing tokens to reading tokens, denoting a new firing of the actor, and (2) whenever a state-reset event occurs.

\footnotetext{
${ }^{4}$ In existing systems, such timestamps are often the only information available and thus are also used to second-guess other properties such as token and object dependencies.

${ }^{5}$ We use a single state-reset event as opposed to separate events for marking the start and end of a transaction.
} 


\begin{tabular}{|c|c|c|c|}
\hline$E_{l o c}$ & $E_{t y p}$ & $E_{t o k}$ & $E_{f i r}$ \\
\hline $\mathrm{p}_{0}$ & $\mathrm{w}$ & $\mathrm{t}_{1}$ & 1 \\
\hline $\begin{array}{l}\cdots \\
\mathrm{p}_{0}\end{array}$ & $\mathrm{w}$ & $\mathrm{t}_{18}$ & \\
\hline$A_{1}$ & $\mathrm{~s}$ & - & \\
\hline $\mathrm{p}_{1}$ & $\mathrm{r}$ & $\mathrm{t}_{1}$ & \\
\hline$\cdots$ & & & \\
\hline $\mathrm{p}_{1}$ & $\mathrm{r}$ & $\mathrm{t}_{7}$ & \\
\hline $\mathrm{p}_{2}$ & $\mathrm{w}$ & $\mathrm{t}_{19}$ & \\
\hline$A_{1}$ & $\mathrm{~s}$ & - & \\
\hline $\mathrm{p}_{1}$ & $\mathrm{r}$ & $\mathrm{t}_{8}$ & \\
\hline$\ldots$ & & & \\
\hline $\mathrm{p}_{1}$ & $\mathrm{r}$ & $\mathrm{t}_{16}$ & \\
\hline $\mathrm{p}_{2}$ & $\mathrm{w}$ & $\mathrm{t}_{20}$ & \\
\hline
\end{tabular}

\begin{tabular}{|c|c|c|c|}
\hline$E_{l o c}$ & $E_{t y p}$ & $E_{t o k}$ & $E_{\text {fire }}$ \\
\hline $\mathrm{A}_{1}$ & $\mathrm{~s}$ & - & 3 \\
\hline $\mathrm{p}_{1}$ & $\mathrm{r}$ & $\mathrm{t}_{17}$ & 3 \\
\hline $\mathrm{p}_{1}$ & $\mathrm{r}$ & $\mathrm{t}_{18}$ & 3 \\
\hline $\mathrm{p}_{2}$ & $\mathrm{w}$ & $\mathrm{t}_{21}$ & 3 \\
\hline $\mathrm{A}_{1}$ & $\mathrm{~s}$ & - & 4 \\
\hline $\mathrm{A}_{2}$ & $\mathrm{~s}$ & - & 1 \\
\hline $\mathrm{p}_{3}$ & $\mathrm{r}$ & $\mathrm{t}_{19}$ & 1 \\
\hline $\mathrm{p}_{4}$ & $\mathrm{w}$ & $\mathrm{t}_{22}$ & 1 \\
\hline $\mathrm{A}_{2}$ & $\mathrm{~S}$ & - & 2 \\
\hline $\mathrm{p}_{3}$ & $\mathrm{r}$ & $t_{20}$ & 2 \\
\hline $\mathrm{p}_{4}$ & $\mathrm{w}$ & $\mathrm{t}_{23}$ & 2 \\
\hline $\mathrm{A}_{2}$ & $\mathrm{~s}$ & - & 3 \\
\hline $\mathrm{p}_{3}$ & $r$ & $\mathrm{t}_{21}$ & 3 \\
\hline
\end{tabular}

\begin{tabular}{cccc}
$E_{l o c}$ & $E_{\text {typ }}$ & $E_{\text {tok }}$ & $E_{\text {fire }}$ \\
\hline $\mathrm{A}_{2}$ & $\mathrm{~s}$ & - & 4 \\
$\mathrm{~A}_{3}$ & $\mathrm{~s}$ & - & 1 \\
$\mathrm{p}_{5}$ & $\mathrm{r}$ & $\mathrm{t}_{22}$ & 1 \\
$\mathrm{p}_{6}$ & $\mathrm{w}$ & $\mathrm{t}_{24}$ & 1 \\
$\mathrm{p}_{6}$ & $\mathrm{w}$ & $\mathrm{t}_{25}$ & 1 \\
$\mathrm{p}_{6}$ & $\mathrm{w}$ & $\mathrm{t}_{26}$ & 1 \\
$\mathrm{~A}_{3}$ & $\mathrm{~s}$ & - & 2 \\
$\mathrm{p}_{5}$ & $\mathrm{r}$ & $\mathrm{t}_{23}$ & 2 \\
$\mathrm{p}_{6}$ & $\mathrm{w}$ & $\mathrm{t}_{27}$ & 2 \\
$\mathrm{p}_{6}$ & $\mathrm{w}$ & $\mathrm{t}_{28}$ & 2 \\
$\mathrm{~A}_{3}$ & $\mathrm{~s}$ & - & 3 \\
$\mathrm{~A}_{4}$ & $\mathrm{~s}$ & - & 1 \\
$\mathrm{p}_{7}$ & $\mathrm{r}$ & $\mathrm{t}_{24}$ & 1
\end{tabular}

\begin{tabular}{|c|c|c|c|}
\hline$E_{l o c}$ & $E_{t y p}$ & $E_{\text {tok }}$ & $E_{f i r}$ \\
\hline $\mathrm{p}_{7}$ & $\mathrm{r}$ & $\mathrm{t}_{25}$ & 1 \\
\hline $\mathrm{p}_{7}$ & $\mathrm{r}$ & $\mathrm{t}_{26}$ & \\
\hline $\mathrm{p}_{8}$ & $\mathrm{w}$ & $\mathrm{t}_{29}$ & \\
\hline $\mathrm{A}_{4}$ & $\mathrm{~s}$ & - & \\
\hline $\mathrm{p}_{7}$ & $\mathrm{r}$ & $\mathrm{t}_{27}$ & \\
\hline $\mathrm{p}_{7}$ & $\mathrm{r}$ & $\mathrm{t}_{28}$ & 2 \\
\hline $\mathrm{p}_{8}$ & $\mathrm{w}$ & $\mathrm{t}_{30}$ & \\
\hline $\mathrm{A}_{4}$ & $\mathrm{~s}$ & - & \\
\hline $\mathrm{p}_{9}$ & $\mathrm{r}$ & $\mathrm{t}_{29}$ & 1 \\
\hline $\mathrm{p}_{9}$ & $\mathrm{r}$ & $\mathrm{t}_{30}$ & 1 \\
\hline
\end{tabular}

Fig. 2. The event log for a run of the example workflow in Fig. 1

\subsection{Complex Workflow Transactions}

In the example event log of Figure 2, state-reset events denote "sub-runs", i.e., independent actor firings operating on sets of associated data. Note that for the particular event log shown, state reset events occur exactly at read/write transitions (i.e., after write events immediately followed by read events) 6 However, for more complex workflows and actors, read/write transitions alone will not determine state-reset events, and more complex event patterns will be required to accurately describe data dependencies. Figure 3 gives four cases in which read/write transitions do not imply actor transaction boundaries, thus requiring more complex uses of state-reset events.

Figure 3(a) shows an actor $A_{1}$ that computes a sequence of running temperature averages $\left(\mathrm{ta}_{n}\right)$ from a series of input temperature readings $\left(\mathrm{t}_{m}\right)$, along with a corresponding event log for an example run. Each average reading is dependent on all temperature readings received since the most recent state-reset of the actor (e.g., at midnight each night). In the example event log, token $t_{24}$ depends on tokens $t_{1}-t_{24}$, while $t_{25}$ is dependent only on $t_{25}$. Note that assuming that an implicit state-reset follows each write event would be incorrect, because this would imply that each temperature average depended only on the latest temperature reading received, rather than all temperature readings received so far during a particular round of firings.

Figure 3(b) illustrates the necessity of recording state-reset events for a filtering actor. In this example, a series of protein structures are input to actor $A_{2}$, and only those structures meeting a minimum resolution requirement are output (though carried by new tokens). All other input protein structures are discarded by the actor. Thus, in the example event log, of the first six structures received by $\mathrm{A}_{2}$, only three are output. Because the state-reset events are recorded,

${ }^{6}$ Note that state-reset events are still necessary in this example to mark the beginning and end of the actor firing/round. 
(a) Compute running daily average temperature
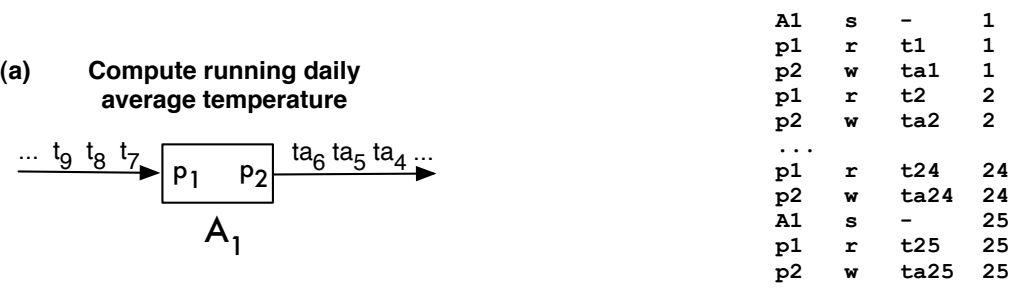

(b)

Filter PDB files

by resolution

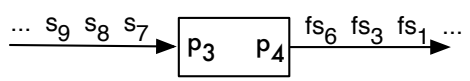

$\mathrm{A}_{2}$

$\begin{array}{llll}\text { A2 } & \text { s } & - & 1 \\ \text { p3 } & \text { r } & \text { s1 } & 1 \\ \text { p4 } & \text { w } & \text { fs1 } & 1 \\ \text { A2 } & \text { s } & - & 2 \\ \text { p3 } & \text { r } & \text { s2 } & 2 \\ \text { A2 } & \text { s } & - & 3 \\ \text { p3 } & \text { r } & \text { s3 } & 3 \\ \text { p4 } & \text { w } & \text { fs3 } & 3 \\ \text { A2 } & \text { s } & - & 4 \\ \text { p3 } & \text { r } & \text { s4 } & 4 \\ \text { A2 } & \text { s } & - & 5\end{array}$

(c)

Iteratively search for most parsimonious phylogenetic trees

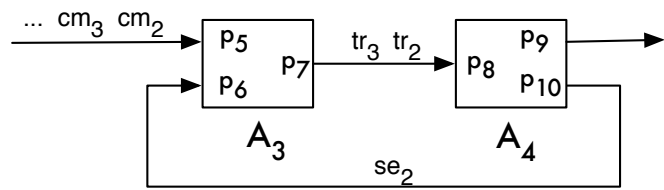

(d) Predict protein $2^{\circ}$ structure using sliding window

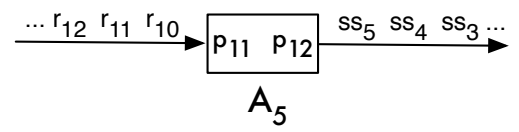

$\begin{array}{llll}\text { A5 } & \text { s } & - & 1 \\ \text { p11 } & r & r 1 & 1 \\ \text { p11 } & r & r 2 & 1 \\ \text { P11 } & & & \\ \text { p11 } & r & r 5 & 1 \\ \text { p12 } & \text { w } & \text { ss1 } & 1 \\ \text { A5 } & \text { s } & - & 2 \\ \text { p11 } & r & r 2 & 2 \\ \text { p11 } & r & r 3 & 2 \\ \text { p1 } & & & \\ \text { p11 } & r & r 6 & 2 \\ \text { p12 } & \text { w } & \text { ss2 } & 2 \\ \text { A12 } & \text { s } & - & 3 \\ \text { p11 } & r & r 3 & 3 \\ \text { p11 } & r & r 4 & 3\end{array}$

Fig. 3. Four distinct types of actors requiring complex state-reset event behavior

however, it is clear, e.g., that output token $\mathrm{fs}_{3}$ depends only on input token $\mathrm{s}_{3}$, and not on $\mathrm{s}_{2}$, even though no write event separates the read events for $\mathrm{s}_{2}$ and $\mathrm{s}_{3}$.

Figure 3(c) illustrates the more general case where an actor reuses only some of the data received during a previous firing. In this example, the tree inference actor $A_{3}$ requires a random number seed to initiate a search for maximally 
parsimonious phylogenetic trees. Since any particular firing of the actor is not guaranteed to find all of the most parsimonious trees, the actor must be fired iteratively for a particular matrix of phylogenetically informative characters, using a distinct seed on each iteration. Actor $A_{4}$ collects the trees inferred by $A_{3}$ and provides the seeds needed by $A_{3}$ until a sufficient number of trees have been inferred. The RWS model allows each tree inferred in this way to be associated not only with the character matrix from which it was derived, but also with the particular random number seed used by $\mathrm{A}_{3}$ to discover the tree. The sample event $\log$ illustrates how this works. Actor $A_{3}$ raises an ' $\mathrm{s}$ ' event prior to receiving each seed, and on receiving that seed declares that it re-reads the character matrix used previously along with the new seed. Thus, it is clear that while trees $\operatorname{tr}_{1}-\operatorname{tr}_{5}$ all depend on character matrix $\mathrm{cm}_{1}$, only $\mathrm{tr}_{4}$ and $\mathrm{tr}_{5}$ were derived using seed $\mathrm{se}_{2}$.

Finally, Figure 3(d) illustrates the requirements for recording the provenance of an actor operating on a sliding window of data. Actor $A_{5}$ predicts the secondary structure of a protein, residue by residue, based on the types of residues (i.e., amino acids) within a contiguous segment of the protein chain. In this case the RWS model allows the actor to raise an 's' event after writing each output token. The actor then re-reads all tokens except the first token in the current window, along with the next token available on the input, before computing its next output 7

\subsection{Dependency Graphs}

Using the RWS model, we are able to infer from the event log the token dependency graph. That is, for each token $t$, we can know which parent tokens $\left\{t_{1}, \ldots, t_{k}\right\}$ directly contributed to the production of $t$ (as the result of an actor firing). As an example, in the upper left of Fig. 4, $\left\{t_{1}, \ldots, t_{7}\right\}$ are parent tokens of $t_{19}$. Conversely, $t_{22}$ is the parent of $t_{24}, t_{25}, t_{26}$. The following Datalog program illustrates how the token dependency graph can be computed from the event log. The event relation corresponds to the event log and the actor relation contains a mapping from ports to their corresponding actors.

$$
\begin{aligned}
& \text { depends-on }\left(T_{1}, T_{2}\right):-\operatorname{event}\left(P_{1}, w, T_{1}, C_{1}\right), \operatorname{event}\left(P_{2}, r, T_{2}, C_{2}\right) \text {, } \\
& \operatorname{actor}\left(P_{1}, A\right), \operatorname{actor}\left(P_{2}, A\right), \operatorname{reset}\left(A, C_{b}, C_{e}\right) \text {, } \\
& C_{b} \leq C_{2} \leq C_{1}<C_{e} \text {. } \\
& \operatorname{reset}\left(A, C_{b}, C_{e}\right):-\operatorname{event}\left(A, s,{ }_{-}, C_{b}\right), \operatorname{event}\left(A, s,{ }_{-}, C_{e}\right), C_{b}<C_{e} \text {, } \\
& \neg \text { reset-between }\left(A, C_{b}, C_{e}\right) \text {. } \\
& \text { reset-between }\left(A, C_{b}, C_{e}\right):-\operatorname{event}\left({ }_{-},-,-, C_{b}\right), \operatorname{event}\left({ }_{-},-,-, C_{e}\right) \text {, } \\
& \text { event }(A, s, X, C), C_{b}<C<C_{e} \text {. }
\end{aligned}
$$

We say that $T_{1}$ depends on $T_{2}$ whenever depends-on $\left(T_{1}, T_{2}\right)$ is true.

In addition to the token dependency graph, we are also able to infer the object dependency graph using the RWS model. Object dependencies describe user data lineage, and are crucial for our "user-oriented" queries. For example, the middle

7 The RWS model could be optimized for cases where actors forget only a small fraction of previously read tokens during each firing by introducing an explicit 'forget' event. 


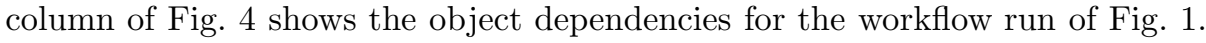
Note that the object dependency graph differs slightly from the token dependency graph. Object dependency graphs can be computed from corresponding token dependency graphs and token-object mappings.
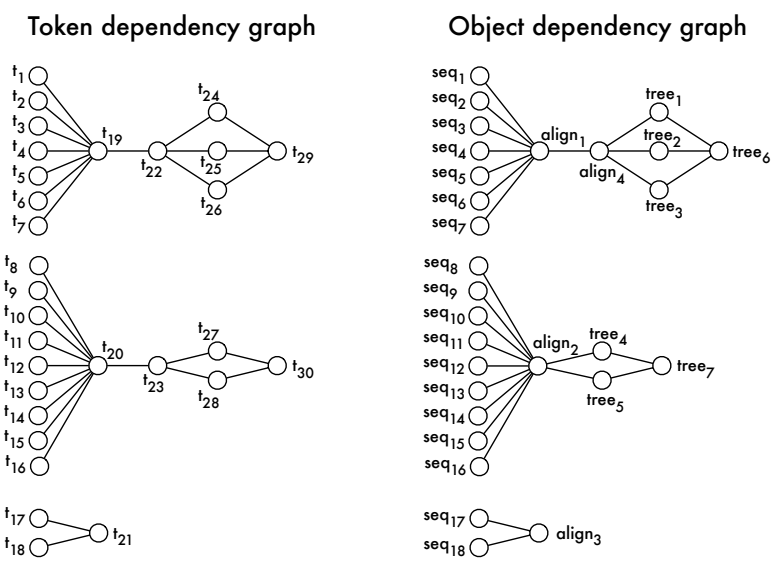

Invocation dependency graph
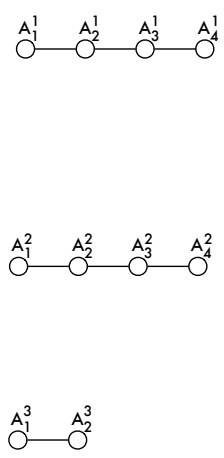

Fig. 4. Token, object, and actor invocation graphs for our example phylogenetics workflow. Dependencies are shown from left to right. Note that all but one of the tokenobject mappings can be inferred from the graph structures; tokens $t_{20}$ and $t_{23}$ both map to the object align $_{2}$.

Finally, actor-invocation dependency graphs can also be inferred directly from event logs in the RWS model. In particular, this graph can be built from statereset events in the event $\log$ such that an actor invocation $A_{2}^{j}$ depends on another actor invocation $A_{1}^{i}$ whenever $A_{2}^{j}$ reads a token that is written by $A_{1}^{i}$. Note that here, "invocation" refers to a firing round. It should be clear that all of the information stored in the event table is required to reconstruct these token, object, and invocation dependency graphs for a workflow trace. In particular, if state-reset events are not taken into account, each token written by an actor will (incorrectly) appear to depend on all previous tokens read during prior firing rounds of the actor: e.g., in the absence of state-reset events, $\mathrm{t}_{21}$ would be connected to tokens $t_{1}$ to $t_{18}$ in the token dependency graph of Fig. 4 ,

\section{Querying Workflow Traces}

A wide range of scientifically relevant questions can be answered using the provenance model described above. To make access to event logs more convenient, we introduce the following primitive operations, which can be implemented, e.g., as relational selections over the event log. The writer $(t)$ and reader $(t)$ operations return the ports that a token $t$ was written to and read from, respectively (a token is written to a port exactly once, but can be read multiple times). The 
token-parents $(t)$ and token-children $(t)$ operations return the set of direct token dependencies for a token $t$, while token-ancestors $(t)$ and token-descendents $(t)$ are their transitive closures. The siblings $(t)$ operation returns the tokens with the same direct dependencies as $t$; e.g., because actor A3 can infer multiple trees from an alignment, given one of these trees, siblings returns the other trees computed from the same alignment. The $\operatorname{origin}(o)$ and death(o) operations return the first and last tokens in the trace that refer to the object o; e.g., the origin and death operations can be used to determine that the alignment object align $n_{2}$ originated with token $t_{20}$ (written by actor $A 1$ ) and terminated with token $t_{23}$ (written by actor $\mathrm{A} 2$ ).

The following examples illustrate how the provenance operations can be combined to answer concrete questions of interest to a scientist using the workflow in Figure 1, For each high-level question below, we define a corresponding parameterized query using set-comprehension syntax 8 , along with the actual results for the event $\log$ given in Figure 2 Below, we use $\mathcal{W}$ to denote the workflow graph (in Figure 1) and $\mathcal{T}$ for the corresponding trace.

- What DNA sequences were input to the workflow? This is one of the first questions a scientist might ask about the workflow run. Given an object type $\$ c$, the paramaterized query

$$
q_{1}(\$ \mathrm{c}):=\{o \mid t \in \operatorname{tokens}(\mathcal{T}) \wedge \operatorname{writer}(t) \in \operatorname{in}(\mathcal{W}) \wedge \operatorname{object}(t)=o \wedge \$ \mathrm{c} \in \operatorname{types}(o)\},
$$

returns the set of objects of type $\$ c$ that were input to the workflow run. For our example trace, $q_{1}$ (SEQUENCE) returns the objects seq to seq $_{18}$. The expression $t \in \operatorname{tokens}(\mathcal{T})$ selects a token from the trace, the expression writer $(t) \in \operatorname{in}(\mathcal{W})$ checks that the token was written by an input port of the workflow $\mathcal{W}$, the expression object $(t)=o$ obtains the object associated with $t$, and the expression $\$ c \in$ types $(o)$ verifies that $o$ has $\$ c$ as a type.

- What phylogenetic trees were output by the workflow? This is another basic question that a scientist might initially ask after a run. Given the query

$$
q_{2}(\$ c):=\{o \mid t \in \operatorname{tokens}(\mathcal{T}) \wedge \operatorname{reader}(t) \in \operatorname{out}(\mathcal{W}) \wedge \operatorname{object}(t)=o \wedge \$ \mathrm{c} \in \operatorname{types}(o)\},
$$

the expression $q_{2}$ (TREE) returns the objects tree ${ }_{6}$ and tree .

- What phylogenetic trees (intermediate or final) were created by the workflow? This question requests both intermediate as well as final data products of a run. Given the query

$$
q_{3}(\$ c):=\{o \mid t \in \operatorname{tokens}(\mathcal{T}) \wedge \operatorname{writer}(t) \notin \operatorname{in}(\mathcal{W}) \wedge \operatorname{object}(t)=o \wedge \$ c \in \text { types }(o)\},
$$

the expression $q_{3}$ (TREE) returns all tree objects of Figure 4. Note that the expression writer $(t) \notin i n(W)$ ensures that the returned trees were not given as input to the workflow.

\footnotetext{
${ }^{8}$ Queries could also be defined in Datalog or in query languages for graphs or semi-
} structured data. 
- What actor created this phylogenetic tree? The following query returns the actors that first wrote the given object $\$$ o:

$$
q_{4}(\$ 0):=\{a \mid t \in \operatorname{origin}(\$ 0) \wedge \operatorname{actor}(\operatorname{writer}(t))=a\} .
$$

The query returns $A_{3}$ for tree $e_{1}$ to tree ${ }_{5}$, and $A_{4}$ for tree ${ }_{6}$ and tree ${ }_{7}$. This question is of particular interest for workflows that employ multiple approaches for inferring phylogenetic trees.

- Which phylogenetic trees were directly used to compute this consensus tree? This question (i.e., what is this tree the "consensus" of?) asks for the intermediate data products supplied to the actor producing a particular workflow output. Given the query

$$
\begin{aligned}
& q_{5}(\$ c, \$ 0):=\left\{o^{\prime} \mid t \in \operatorname{origin}(\$ \mathrm{o}) \wedge t^{\prime} \in \text { token-parents }(t) \wedge \operatorname{object}(t)=o^{\prime} \wedge\right. \\
& \left.\$ c \in \operatorname{types}\left(o^{\prime}\right)\right\} \text {, }
\end{aligned}
$$

the expression $q_{5}\left(\right.$ TREE $\left._{\text {, tree }}\right)$ returns tree to $_{1}$ tree $_{3} ;$ and $q_{5}($ TREE, tree $)$ returns tree $_{4}$ to tree ${ }_{5}$.

- What sequences input to the workflow does this consensus tree depend on? This question illustrates how a workflow output can be related to the particular workflow inputs from which it was derived. Given the query

$$
\begin{aligned}
q_{6}(\$ \mathrm{c}, \$ \mathrm{o}):=\left\{o^{\prime} \mid t \in \operatorname{\operatorname {origin}}(\$ \mathrm{o})\right. & \wedge t^{\prime} \in \text { token-ancestors }(t) \wedge \operatorname{writer}\left(t^{\prime}\right) \in \operatorname{in}(\mathcal{W}) \wedge \\
\operatorname{object}\left(t^{\prime}\right)=o^{\prime} & \left.\wedge \$ \mathrm{c} \in \operatorname{types}\left(o^{\prime}\right)\right\}
\end{aligned}
$$

the expression $q_{6}\left(\right.$ SEQUENCE, tree 6 ) returns $\mathrm{seq}_{1}$ to $\mathrm{seq}_{7}$, and the expression $q_{6}\left(\right.$ Sequence, $\left._{\text {tree }}\right)$ returns seq to $_{8}$ seq $_{16}$.

- Which input sequences were not used to derive any output consensus trees? Here we are interested in whether there are any workflow inputs without corresponding workflow outputs. Such inputs may be considered the workflow equivalent of "phantom lineages" 23]. Given an input type $\$ c_{i n}$ and output type $\$ \mathrm{c}_{\text {out }}$, the query

$$
\begin{aligned}
q_{7}\left(\$ \mathrm{c}_{\text {in }}, \$ \mathrm{c}_{\text {out }}\right):=\{0 \mid & t \in \operatorname{tokens}(\mathcal{T}) \wedge \text { writer }(t) \in \operatorname{in}(\mathcal{W}) \wedge \operatorname{object}(t)=o \wedge \\
& \$ \mathrm{c}_{\text {in }} \in \operatorname{types}(o) \wedge\left\{t^{\prime} \mid t^{\prime} \in \operatorname{token} \text {-descendents }(t) \wedge\right. \\
& \text { reader } \left.\left.\left(t^{\prime}\right) \in \operatorname{out}(\mathcal{W}) \wedge c_{\text {out }} \in \operatorname{types}\left(\operatorname{object}\left(t^{\prime}\right)\right)\right\}=\emptyset\right\},
\end{aligned}
$$

returns the objects input to the workflow that do not produce any workflow outputs; e.g., the expression $q_{7}$ (SEQUENCE, TREE) returns the sequences $\operatorname{seq}_{17}$ and $\mathrm{seq}_{18}$. The query first finds workflow input tokens $t$ that refer to objects of type $\$ \mathrm{c}_{i n}$, and then checks (via a subquery) to make sure that $t$ has no output tokens with objects of the type $\$ \mathrm{c}_{\text {out }}$.

- What was the sequence alignment used in the process of inferring this tree? This question requests the key intermediate data object used in producing a workflow result. A researcher may wish to examine the alignment to assess the reliability of the results, or reuse the alignment in another workflow. 
Given the query

$$
\begin{aligned}
q_{8}(\$ c, \$ o):=\left\{o^{\prime} \mid\right. & t \in \operatorname{origin}(\$ o) \wedge t^{\prime} \in \text { token-ancestors }(t) \wedge \operatorname{object}\left(t^{\prime}\right)=o^{\prime} \wedge \\
& \$ c \in \operatorname{types}\left(o^{\prime}\right) \wedge\left\{t^{\prime \prime} \mid t^{\prime \prime} \in \text { token-descendents }\left(t^{\prime}\right) \wedge\right. \\
& \left.\left.\$ \mathrm{c} \in \operatorname{types}\left(\operatorname{object}\left(t^{\prime}\right)\right)\right\}=\emptyset\right\},
\end{aligned}
$$

the expression $q_{8}\left(\right.$ AlignMENT, tree $\left._{6}\right)$ returns the sequence alignment align 4 , and $q_{8}\left({\left.\text { Alignment }, \text { tree }_{7}\right) \text { returns the sequence alignment align }}_{2}\right.$. The subquery above ensures that the object $o^{\prime}$ is the alignment directly used to infer the tree.

- What actors were involved in creating this tree? This question may be used, e.g., when writing the methods section of a publication to cite the employed methods and implementations. Given the query

$$
\begin{aligned}
q_{9}(\$ 0):= & \{a \mid t \in \operatorname{origin}(\$ 0) \wedge \operatorname{actor}(\text { writer }(t))=a\} \cup \\
& \left\{a \mid t \in \operatorname{origin}(\$ 0) \wedge t^{\prime} \in \text { token-ancestors }(t) \wedge \operatorname{actor}\left(\text { writer }\left(t^{\prime}\right)\right)=a\right\},
\end{aligned}
$$

the expression $q_{9}\left(\right.$ tree $\left._{6}\right)$ returns actors $\mathrm{A} 1$ to $\mathrm{A} 4$.

- Which actors did not produce any output for input derived from this input sequence? This question provides an explanation for the phantom lineages revealed by $q_{7}$ above:the query

$$
\begin{gathered}
q_{10}(\$ o):=\left\{a \mid t \in \operatorname{origin}(o) \wedge t^{\prime} \in \text { token-descendents }(t) \wedge \text { token-children }\left(t^{\prime}\right)=\emptyset\right. \\
\left.\wedge \operatorname{actor}\left(\operatorname{reader}\left(t^{\prime}\right)\right)=a\right\}
\end{gathered}
$$

The expressions $q_{10}\left(\mathrm{seq}_{17}\right)$ and $q_{10}\left(\mathrm{seq}_{17}\right)$ both return actor A2, indicating that this actor did not forward a refined sequence alignment of these two sequences to actor A3. This result is reasonable since no informative phylogenetic trees may be inferred from only two taxa.

\section{Conclusion}

Tracking provenance is an important aspect of scientific workflow systems. In this paper, we have focused primarily on the problem of tracking data lineage within scientific workflow runs, for the purpose of providing an accurate provenance record for answering "scientific" (i.e., user-oriented) provenance queries.

The problem of data lineage has been widely studied in the database community 58 2 23]. However, the primary focus has been on transformations of data items expressed as database queries. As noted in [11, current provenance approaches for workflow systems (e.g., 242518) record various kinds of metadata related to provenance. Despite these developments, however, little support exists in current systems to allow end-users to query provenance information in scientifically meaningful ways, in particular when advanced workflow execution models go beyond simple DAGs (as in process networks).

We have shown that a simple provenance model, based on read, write, and state-reset events, is expressive enough to capture many relevant science-oriented provenance use cases. These use cases become queries against suitable views on 
top of the event log. Our approach also marks the beginnings of a use-case and computation-model driven approach to provenance schema design. Using our framework, it is now meaningful to ask whether a provenance schema can handle specific use cases, since the latter become queries over the former.

As future work we intend to extend our approach to support a wider array of operations, e.g., so-called "smart re-runs" (a workflow system requirement in 16] $)^{9}$ and crash recovery, and to extend our current Prolog-based prototype to provide direct support (including query user interfaces) for our provenance model within KEPLER. We are also developing methods to optimize our approach to reduce the size of event logs for actors whose behaviors are similar to sliding window operators (e.g., by introducing a "forget" event), and to support subworkflows within KEPLER (i.e., composite actors), e.g., by inferring in a bottom-up fashion the appropriate state-reset events for the composite actor via the state-reset events of subsumed actors and the corresponding workflow graph.

\section{References}

1. D. Berry, P. Buneman, M. Wilde, and Y. Ioannidis, editors. e-Science Workshop on Data Provenance and Annotation, National e-Science Centre, Edinburgh, December 2003.

2. D. Bhagwat, L. Chiticariu, W. C. Tan, and G. Vijayvargiya. An annotation management system for relational databases. In Proc. of $V L D B, 2004$.

3. R. Bose and J. Frew. Lineage retrieval for scientific data processing: A survey. ACM Computing Surveys, 37(1):1-28, 2005.

4. P. Buneman and I. Foster, editors. Workshop on Data Derivation and Provenance, Chicago, October 2002.

5. P. Buneman, S. Khanna, and W. C. Tan. Why and where: A characterization of data provenance. In Proc. of ICDT, volume 1973 of LNCS, 2001.

6. S. P. Callahan, J. Freire, E. Santos, C. E. Scheidegger, C. T. Silva, and H. T. Vo. Managing the evolution of dataflows with vistrails. In IEEE Workshop on Workflow and Data-Flow for Scientific Applications (SciFlow), 2006.

7. J. Castresana. Selection of conserved blocks from multiple alignments for their use in phylogenetic analysis. Mol. Biol. Evol., 17:540-552, 2000.

8. Y. Cui and J. Widom. Lineage tracing for general data warehouse transformations. In $V L D B, 2001$.

9. Y. Cui, J. Widom, and J. Wiender. Tracing the lineage of view data in a warehousing environment. ACM TODS, 25(2), 2000.

10. E. Deelman, J. Blythe, Y. Gil, C. Kesselman, G. Mehta, S. Patil, M.-H. Su, K. Vahi, and M. Livny. Pegasus: Mapping scientific workflows onto the grid. In Proc. of the European Across Grids Conference, 2004.

11. C. Goble. Position statement: Musings on provenance, workflow and (semantic web) annotations for bioinformatics. In Buneman and Foster 4 .

12. G. Kahn and D. B. MacQueen. Coroutines and networks of parallel processes. In Proc. of the IFIP Congress, 1977.

${ }^{9}$ Specialized provenance systems for smart re-runs exist already 6 . 
13. E. A. Lee and D. Messerschmitt. Static scheduling of synchronous data flow programs for digital signal processing. IEEE Transactions on Computers, C-36, 1987.

14. E. A. Lee and S. Neuendorffer. Actor-oriented models for codesign: Balancing reuse and performance. In Formal Methods and Models for System Design. Kluwer, 2004 .

15. E. A. Lee and T. M. Parks. Dataflow process networks. Proc. of the IEEE, 83(5), 1995.

16. B. Ludäscher, I. Altintas, C. Berkley, D. Higgins, E. Jaeger, M. Jones, E. A. Lee, J. Tao, and Y. Zhao. Scientific workflow management and the Kepler system. Concurrency and Computation: Practice 63 Experience, 2005.

17. L. Moreau, O. Rana, and D. Walker. Provenance aware service-oriented architecture (pasoa). pasoa.org, 2006.

18. T. M. Oinn, M. Addis, J. Ferris, D. Marvin, M. Senger, R. M. Greenwood, T. Carver, K. Glover, M. R. Pocock, A. Wipat, and P. Li. Taverna: A tool for the composition and enactment of bioinformatics workflows. Bioinformatics, 20(17), 2004.

19. PHYLIP Phylogeny Inference Package. http://evolution.gs.washington.edu/ phylip.html

20. Y. Simmhan, B. Plale, and D. Gannon. A survey of data provenance in e-science. SIGMOD Record, 34(3):31-36, September 2005.

21. D. Thain, T. Tannenbaum, and M. Livny. Distributed computing in practice: The Condor experience. Concurrency - Practice and Experience, 17(2-4), 2005.

22. J. D. Thompson, D. G. Higgins, and T. J. Gibson. CLUSTAL W: Improving the sensitivity of progressive multiple sequence alignment through sequence weighting, position specific gap penalties and weight matrix choice. Nucleic Acids Res., 22:4673-80, 1994.

23. J. Widom. Trio: A system for integrated management of data, accuracy, and lineage. In Conference on Innovative Data Systems Research (CIDR), 2005.

24. S. Wong, S. Miles, W. Fang, P. Groth, and L. Moreau. Provenance-based validation of e-science experiments. In $I S W C, 2005$.

25. J. Zhao, C. Goble, R. Stephens, and S. Bechhofer. Linking and browsing provenance logs for e-science. In ICSNW, 2004. 